\title{
Las controversias sociocientíficas como herramienta didáctica para el desarrollo de la alfabetización científica
}

\section{Using socioscientific issues as an educational tool to develop scientific literacy}

\author{
Naira Díaz Moreno. \\ Universidad de Almería. \\ nairadia@ual.es \\ Ester Caparrós Martín. \\ Universidad de Málaga. \\ ester.caparros@uca.es

\section{J. Eduardo Sierra Nieto.} \\ Universidad de Málaga. \\ eduardo.sierra@uca.es
}

\section{RESUMEN.}

La desconexión existente entre la ciencia que es llevada a cabo por los científicos y la ciencia escolar es una realidad que se refleja no sólo en diferentes informes sino también en el creciente desinterés del alumnado por las cuestiones científicas. En este artículo nos planteamos la formación en competencias científicas y, más concretamente, la adquisición de la alfabetización científica a través de las cuestiones o controversias sociocientíficas (SSI). Las SSI acercan la Ilamada "ciencia en construcción" a la realidad del aula, despertando el interés del alumnado con temas controvertidos científica y socialmente. Estos problemas abiertos nos sirven como contexto para estudiar la ciencia y sobre la ciencia; y constituyen, además, una potente herramienta para alcanzar la denominada ciencias para todos y todas, una de las líneas de investigación más relevante en la enseñanza de las ciencias en los últimos años.

\section{PALABRAS CLAVE.}

Controversias sociocientíficas, alfabetización científica, enseñanza de las ciencias.

\section{ABSTRACT.}

The science that is making by scientists and scholar science are not related. This lack of connection between the two is reflected in different reports and also in the increasing disinterest of students about the science subjects. In this paper, we set out training in scientific competence and, specifically, the achievement of the scientific literacy through socioscientific issues (SSI). SSI bring "science in the making" to the class wakening the interest of the students in themes that are related to science and to society. These open problems are a good context to study science and about the science that at the same time they constitute a powerful tool to develop scientific literacy. Furthermore, during the last years, SSI has constituted one of the more innovative lines of research in science education.

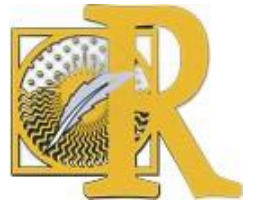




\section{KEY WORDS.}

Socioscientific issues, scientific literacy, science education.

\section{Introducción.}

La educación científica ha sido y sigue siendo una cuestión controvertida, tal y como se ha venido repitiendo en los distintos informes internacionales de evaluación ya conocidos, no sólo en España sino en todos los países de la OCDE (Jiménez, 2012). Del conocido como informe Rocard ${ }^{1}$, publicado en 2007 , se puede extraer como conclusión que los estudiantes perciben la educación científica como irrelevante y difícil, poniendo de manifiesto que existe una conexión entre las actitudes hacia la ciencia y la enseñanza (y el aprendizaje) de ésta. Esto es así hasta el punto de que, como sugiere Pedrinaci et al (2012), urge introducir cambios sustanciales en la enseñanza de las ciencias con la finalidad de alcanzar un relación positiva y fructífera entre actitud y enseñanza.

Esa dificultad en el aprendizaje de las ciencias a la que hace alusión el informe Rocard se puede considerar como una influyente causa en el alto índice de abandono escolar prematuro en la educación secundaria en España -aproximadamente el 30\% del alumnado no termina la ESO, en 2010 a nivel estatal el 28,4\%-, lo que ha sido objeto de múltiples y variadas publicaciones en los últimos años (Jiménez, 2012).

La Quinta Encuesta sobre percepción Social de la Ciencia y la Tecnología en España (FECYT, 2011) indica que el $40,5 \%$ de los encuestados valora como bajo o muy bajo el nivel de educación científica que han recibido y sólo un 10,7\% lo considera alto o muy alto. En esta línea, tanto el informe EURYDICE (2011) como el Informe ENCIENDE (Couso et al., 2011) ponen de manifiesto la problemática que supone el bajo rendimiento del alumnado en Ciencias. Así, coincidimos con Pedrinaci et al (2012) al afirmar que los datos de los citados informes evidencian la existencia de una brecha entre los aprendizajes promovidos por las ciencias en la escuela actualmente y las demandas sociales; o dicho de otra manera, entre educación científica y sociedad. Lo que tratamos de evidenciar es la fisura tan grande que existe entre la manera qué se enseña y se aprende la ciencia en las escuelas y lo que realmente la ciencia significa y es para la sociedad actual. Ariza et al. (2014) sostienen con respecto a estas ideas que:

La educación científica debe de avanzar para resolver estas demandas y para asegurar no solamente la formación de científicos de élite, sino la educación de ciudadanos críticos, que tomen parte en los debates de problemas socio-científicos. Es decir, la educación científica tiene que formar tanto a científicos como a no científicos con el conocimiento, las competencias y los valores necesarios para poder contribuir a las demandas de nuestra sociedad. (p. 109)

Es relativamente fácil argumentar, como señala Lindsay (2011), que actualmente existe una discordancia entre el tipo de ciencia que es percibida por la sociedad y aquella que se continúa reproduciendo en las escuelas ${ }^{2}$; discordancia que también existe entre la ciencia escolar y los encargados de hacer políticas educativas en ciencias. Sabemos que el cambio hacia una nueva concepción y práctica de la educación en ciencias es muy lento; quizás porque el pensamiento dominante de la ciencia, propio de la era industrial y que se caracteriza por la racionalidad y la objetividad, es muy difícil de transformar.

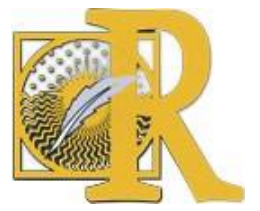


En este sentido, cualquier cambio hacia una concepción más social de la ciencia es entendido como "ligero", en el sentido de "liviano" o poco relevante; además de ser considerado por algunos científicos y profesorado de ciencias como falto de contenido. De ahí que se produzca un rechazo hasta el punto de ver "normal" que desaparezca la asignatura obligatoria de Bachillerato "Ciencias para el Mundo Contemporáneo" -centrada en los contenidos sociocientíficos actuales (biotecnología, salud, medioambiente, entre otros)-.

Siguiendo los planteamientos de Lindsay (opus cit.), podemos destacar dos elementos principales que contribuyen a fomentar y a potenciar esa necesidad de avanzar hacia un cambio en la educación en ciencias. En primer lugar, nos encontramos con el hecho de que a nivel social nos enfrentamos a una serie de temas socio-ambientales controvertidos como son el cambio climático, la biodiversidad, la investigación de células madre, la clonación, la nanotecnología, etc. En segundo lugar, nos hallamos sometidos a la "sobreinformación" a través de los medios de comunicación, sobre todo en lo relativo a dichos temas; cuyo impacto es la presentación continua de información relacionada con la ciencia para los individuos. Es la combinación de estos dos elementos lo que nos lleva a sostener que las y los estudiantes necesitan competencias nuevas, más adecuadas, para que puedan relacionarse con la ciencia de otro modo. Se trata de que las y los estudiantes logren conectar el estudio de las ciencias con los problemas reales que vivimos en nuestra sociedad.

Si la ciudadanía adquiere las capacidades o competencias adecuadas para enfrentarse a estos temas, incluidas las controversias sociocientíficas (de las que nos ocupamos aquí), entonces, se encontrará en una mejor disposición para tomar decisiones con respecto a dichos temas, de manera crítica y efectiva.

Teniendo en cuenta lo planteado hasta el momento, lo que pretendemos abordar en las siguientes líneas es, en primer lugar, las bases de la alfabetización científica como finalidad última en la enseñanza de las ciencias; asumiendo el enfoque de enseñanza de una "ciencia para todos/as" (Couso et al., 2011) como origen de la participación ciudadana en la toma de decisiones de asuntos relacionados con la ciencia y la tecnología, y por tanto, en el ejercicio de la ciudadanía. En segundo lugar, situaremos las controversias sociocientíficas, o lo que es lo mismo, socioscientific issues (a partir de ahora SSI) como herramienta didáctica para alcanzar esta alfabetización científica; respondiendo así a la necesidad de suscitar en el público el debate, el contraste de diferentes puntos de vista, y el posicionamiento ante un problema no resuelto en el ámbito social o científico. En este caso, partimos de la definición que Solbes y Torres (2012) dan de las SSI, entendiéndolas como aquellas controversias sociales que tienen su base en nociones científicas y que además se relacionan con otros campos: sociales, éticos, políticos y ambientales (Jiménez-Aleixandre, 2010). Son cuestiones socialmente vivas que no están decididas ni por la ciencia ni por la sociedad, que están abiertas y suscitan debates y controversias (Legardez y Simonneaux, 2006). Por tanto, tomamos la definición de controversia sociocientífica de Kolstø (2001a), y llamamos controversias sociocientíficas a aquellos problemas relacionados con ciencia y sociedad debido a la compleja relación que existe entre ambas y que normalmente surgen

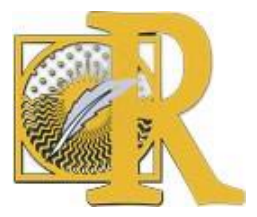


cuando existe una diferencia de opiniones o desacuerdos entre periodistas, ciudadanos y científicos.

Asimismo, señalar que abordamos la formación en competencias científicas a través de las SSI, de manera similar a lo que el planteamiento de problemas abiertos supone para la educación científica en contextos formales; de modo que analizaremos cómo ha evolucionado esta línea de controversias sociocientíficas dentro de la enseñanza de las ciencias y situaremos las tendencias actuales de investigación, siempre dentro de la Didáctica de las Ciencias Experimentales, en relación con las SSI.

\section{Acerca de la alfabetización científica o ciencia para todas y todos.}

La ciencia ha sido considerada "la reina" del siglo XX y todo apunta a que va camino de seguir siéndola en el actual (Castaño et al., 2006). Hoy nadie pone en duda su presencia e importancia en nuestra vida cotidiana, donde los medios de comunicación nos hacen partícipes de noticias y debates sociales relacionados con temas de ciencia y tecnología. Por ello, en los últimos años se ha ido poniendo de relieve la necesidad de ir dotando a los futuros ciudadanos de una formación científica y tecnológica básica.

La rapidez y la trascendencia de los descubrimientos científicos y tecnológicos recientes, como la existencia de las ondas gravitacionales, la vacuna efectiva contra el ébola o la selección de embriones evitando así el gen que desarrolla el cáncer de mama hereditario, por citar algunos, plantean la necesidad de una ciudadanía científicamente culta, que conozca los hechos, pero también funcional, capaz de integrar nuevos conocimientos (Marco-Stiefel, 2003).

Como decimos, en nuestra sociedad, cada día es más notoria la presencia de cuestiones de base científica sobre las que la ciudadanía debe de tener una opinión fundamentada ya que le afecta tanto personal como socialmente (Pedrinaci et al, 2012). La ciencia ha dejado de ser un asunto que compete sólo a los especialistas para convertirse en patrimonio y responsabilidad de todos y todas. Bajo la necesidad de desarrollar una nueva enseñanza de las ciencias surgió el movimiento Ciencia-Tecnología-Sociedad (CTS) a mediados de la década de los 70 que se ha centrado en estudiar la relación existente entre saber ciencia y saber ciudadanía; o mejor, ejercer de científico/a con ejercer de ciudadano/a, destacando así la importancia de situar las ciencias y las tecnologías en su perspectiva social (Aguilar, 2001), y de superar la distancia formada entre formación científica y ciudadanía antes mencionada.

En nuestra opinión estos estudios que relacionan ciencia, tecnología y sociedad, y en concreto, las controversias sociocientíficas que desarrollaremos en el siguiente epígrafe, presentan un gran potencial para formar ciudadanos informados, responsables y capaces de tomar decisiones razonadas y democráticas en la sociedad. El conocimiento científico debe formar parte de la cultura personal, permitiéndonos interpretar la realidad con racionalidad y libertad, y facilitándonos argumentos con la finalidad última de tomar decisiones fundamentadas y críticas en los asuntos relacionados con ciencia y tecnología que nos rodean. 
Siguiendo este propósito de preparar a ciudadanas y ciudadanos, no sólo para estar bien informados, sino para que sean capaces de elaborar sus propias ideas acerca del mundo en el que viven, consideramos primordial que el sistema educativo se replantee lo siguiente: (i) que posibilite una formación adecuada en los términos explicitados; (ii) que facilite las herramientas necesarias para que las y los estudiantes puedan enfrentarse a los asuntos científico-tecnológicos con conciencia y reflexión; (iii) que favorezca las condiciones educativas adecuadas para que el alumnado tome decisiones bien informadas sobre dichos asuntos; (iv) que tome como finalidad última de la enseñanza de las ciencias, la alfabetización científica. Esto último significa que es necesario que la enseñanza de las ciencias responda a las demandas del mundo actual -un mundo cada vez más diverso e interconectado-, facilitando a la ciudadanía el desarrollo de las destrezas y los conocimientos necesarios para que puedan participar activamente y con éxito en la sociedad. Esta alfabetización científica de la que hablamos es importante no solo para aquellos que finalmente lograrán formar parte de la comunidad científica, sino también para todas las personas participes de la sociedad (Couso et al., 2011). En cuanto al significado, el sentido y el uso de la alfabetización científica, en los siguientes apartados nos detendremos brevemente a fin de entender la posición desde la que partimos para abordar las SSI.

\section{1 ¿Qué se entiende por alfabetización científica?}

Sostiene Lindsay (2011), acerca de la noción de alfabetización científica, como ésta hace referencia a la tentativa de avanzar hacia un uso más social de la concepción que habitualmente se sostiene sobre la ciencia; además de explicitar un uso más humano y contemporáneo de la educación científica, tal y como hemos ido señalando hasta ahora. Esta alfabetización científica necesaria para una ciudadanía cada vez más preparada e informada en términos científico-tecnológicos, nos transporta a la "educación científica de base para todas y todos" y que surge como respuesta a las inquietudes con respecto a la enseñanza de las ciencias en los países industrializados (Fourez, 2005).

Sin embargo, aunque la idea de la alfabetización científica parece estar clara en la Comunidad de enseñanza de las ciencias, no existe consenso sobre la definición de este constructo (DeBoer, 2000). La literatura sobre la misma se ha multiplicado en las últimas dos décadas con un verdadero aluvión de definiciones. En este caso, la existencia de esta literatura tan diversa quizás pueda entenderse mejor si se tienen en cuenta las tensiones políticas e intelectuales inherentes a la educación en ciencias (Blanco-García, s/f).

Consideramos relevante, destacar la definición que ofrece Bybee (1997, p. 42) acerca de la alfabetización científica, ya que expresa como ésta:

(...) significa que una persona puede preguntar, hallar o dar respuesta a cuestiones que su curiosidad le plantea diariamente. Significa que una persona es capaz de describir, explicar y predecir fenómenos naturales. La alfabetización científica capacita para leer en la prensa artículos sobre ciencia y para participar en debates sociales sobre la validez de sus conclusiones. La alfabetización científica implica que la persona puede identificar los temas científicos que determinan las decisiones políticas y expresar posiciones informadas científica y tecnológicamente. 
Como podemos observar, el autor ofrece una noción sobre alfabetización científica que está en consonancia con la posición que estamos sosteniendo a lo largo de este texto acerca de la enseñanza de las ciencias; así como, el tipo de competencias en relación a los contenidos y problemas científico-tecnológicos, que deben adquirir las y los estudiantes durante su formación. Con respecto a esto último, nuestro autor (opus cit., p. 68) continúa diciendo que aquello que necesita la ciudadanía científicamente alfabetizada es:

ser capaz de valorar la calidad de la información científica basándose en la fuente de la que procede y en los métodos utilizados para generarla. La alfabetización científica también implica tener la capacidad de valorar los argumentos que se derivan de los hechos establecidos y llegar a conclusiones.

Asimismo, Blanco-López (2004) sostiene como la alfabetización científica engloba, por tanto, un conjunto de saberes, de capacidades o de competencias relevantes para comprender y desenvolverse en el mundo actual. Dicho autor (opus cit.) considera que la finalidad de la enseñanza de las ciencias es lograr que toda la población tenga unos conocimientos científicos que le permita no sólo comprender las noticias relacionadas con ciencia y tecnología -presentes en los medios de comunicación-, sino tomar decisiones y participar activamente en aquellos aspectos relacionadas con temas de naturaleza científico-tecnológicos. Con esta finalidad, se hace más ambiciosa la educación científica ya que el objetivo es llegar a toda la población y no sólo a los futuros estudiantes de ciencias experimentales. Esto muestra un claro reflejo de un cambio social, de un salto que se está consolidando y que afianza la idea de que la ciencia forma parte de la cultura (Martín-Díaz, Gutiérrez y Gómez, 2011).

\subsection{Uso de la alfabetización científica como enfoque curricular.}

Si nos centramos en la idea de alfabetización científica como enfoque curricular es necesario remontarse a finales de los años ochenta donde aconteció tanto un gran fracaso en los currículos científicos, como unos altos índices de desmotivación de las materias científicas por parte del alumnado (Marco-Stiefel, 2001). En este momento, el primer acercamiento a temas que interesaban al alumnado lo lograron los enfoques propuestos bajo el movimiento Ciencia-Tecnología-Sociedad y que fueron plasmados en proyectos curriculares como el SATIS 16-19 (Marco-Stiefel, 1995). A mediados de los años ochenta es cuando aparece la alfabetización científica como objetivo a alcanzar en las próximas décadas, fruto de la publicación de un informe por parte de la Royal Society que fue su punto de partida. Además, en 1992 surgió en Londres la revista Public Understanding of Science $^{3}$-que llevaba el mismo título que el informe-, y que aún continúa planteando el diálogo sobre la alfabetización científica, cubriendo todos los aspectos que se derivan de las interrelaciones entre la ciencia y la sociedad. Otros referentes importantes a nivel anglosajón fueron el Proyecto 2061- Ciencias para todos los americanos, también de 1985, y más recientemente, el Proyecto Beyond 2000 - Science Education for the Future.

Al situar la alfabetización científica dentro del currículum, podemos identificar el papel de dos legítimas, pero potencialmente conflictivas posiciones: (i) la ciencia misma como materia; (ii) las situaciones en las que ésta puede, legítimamente, jugar un papel en otros

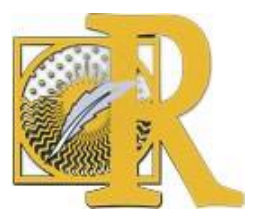


asuntos humanos. Estas dos tendencias han sido las utilizadas para generar componentes en la enseñanza de las ciencias, siendo cada vez más notable en la literatura una polarización existente entre las dos posiciones de defensa que argumentan para llevar estas posturas a los extremos (Roberts, 2007). Hay, por tanto, y según este autor, dos visiones de alfabetización científica: la llamada visión I y la visión II (ver tabla 1).

En cuanto a la visión I, lo que ofrece es una perspectiva de alfabetización científica que mira hacia dentro del canon ortodoxo de las ciencias naturales, lo que significa que se centra solo en los productos y los procesos de la ciencia misma; este es un enfoque que prevé la alfabetización dentro de la ciencia. Por otro lado, está la visión II que deriva su significado de la naturaleza de las situaciones con un componente científico; es decir, aquellas en las que los estudiantes van a encontrarse como ciudadanos, y en la que tiene lugar más consideraciones que sólo las relacionadas con la ciencia misma.

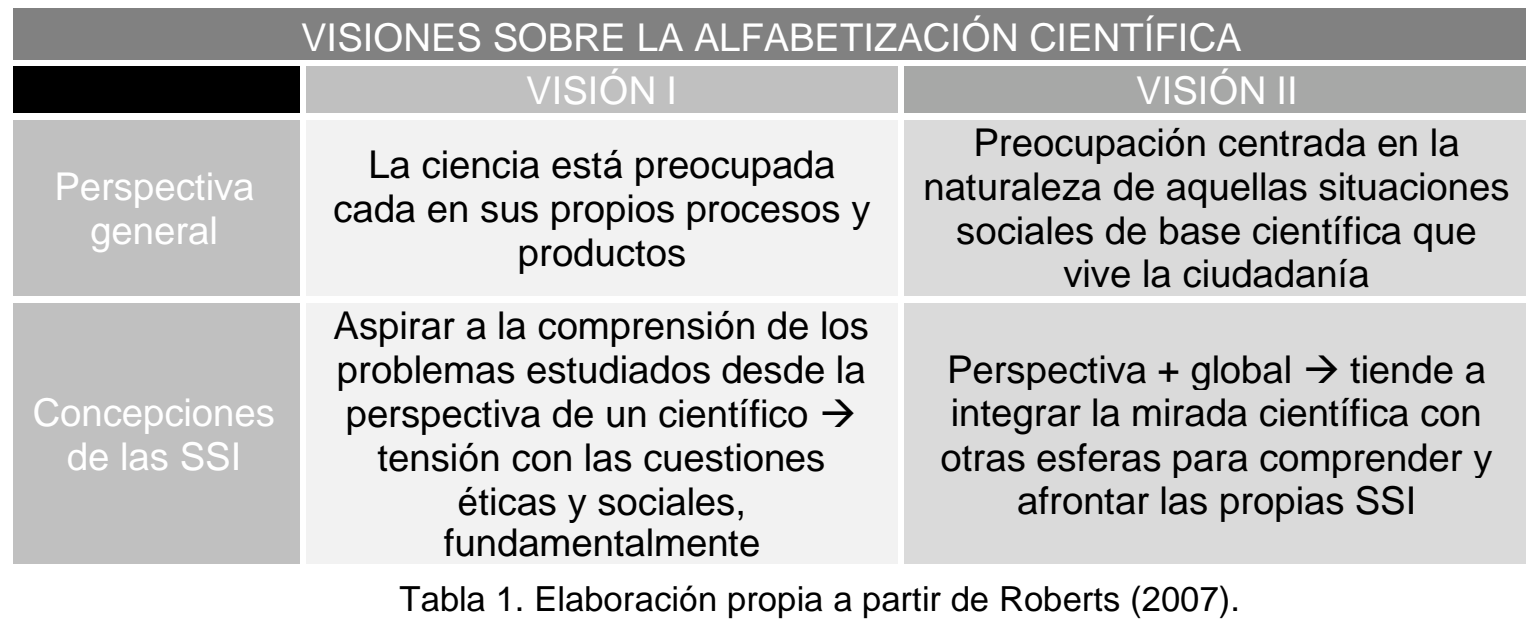

Entre ambas visiones la distinción más evidente tiene que ver con la forma de conceptualizar y experimentar el carácter de las controversias y problemas sociocientíficos. La visión I permite a los estudiantes entender los problemas como un científico lo haría, lo cual proporciona un punto de vista sesgado sobre el tema, relacionado solamente con el conocimiento científico sin tener en consideración otro tipo de cuestiones; como por ejemplo las interacciones que se producen entre ciencia, sociedad e instituciones. Por su parte, la visión II ofrece una mirada más holística; siendo ésta la necesaria para trabajar con controversias sociocientíficas, pues se necesita contemplar otras perspectivas (económicas, políticas, éticas, sociales) para que sean usadas en la reflexión sobre las propias controversias. Así, mientras que un currículum escolar con una visión I puede desarrollarse en ausencia de los discursos de la visión II; la visión II incluye la visión I, algo que no ocurre necesariamente al revés.

Como se puede ir viendo, esta visión II encaja con la concepción más extendida de competencia científica que la OCDE (2006) ha definido a través de las pruebas de PISA, donde dicha competencia se define como "la capacidad de emplear el conocimiento científico para identificar problemas, adquirir nuevos conocimientos, explicar fenómenos científicos y extraer conclusiones basadas en pruebas sobre cuestiones relacionadas con la

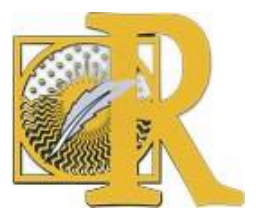


ciencia". Una definición en la que, como antes se apuntó, el conocimiento "de la ciencia" (paralelismo con la visión I) se combina con el conocimiento "sobre la ciencia (paralelismo con la visión II).

Enlazamos así el concepto de alfabetización científica - que se correspondería con la visión II de Roberts (2007) - con el de competencia científica (Pedrinaci et al 2012); y destacamos del término competencia científica la necesidad de priorizar las capacidades que ayudan a la ciudadanía a ejercer sus derechos e integrarse mejor en un mundo globalizado; pese a que según el autor (opus cit.), supera algunos de los inconvenientes que estaban dificultando su aplicación.

Por otra parte, Couso et al. (2011) señalan que el marco de habilidades respecto a la competencia científica se plantea desde una perspectiva de la alfabetización científica, identificando ambas; ya que la competencia científica no solo es importante para los que acaben formando parte de la comunidad científica, sino para la totalidad de los ciudadanos, esto es, como alfabetización científica.

\section{La alfabetización científica para la enseñanza y el aprendizaje de las ciencias.}

La investigación en Didáctica de las Ciencias ha ido señalando en los últimos años la necesidad de avanzar hacia esta alfabetización o competencia científica con el fin de la que ciudadanía disfrute de desarrollo de la ciencia y la tecnología y para la participación en debates públicos (Martínez-Losada, 2010), si bien algunos lo consideraban un mito (Shamos, 1995). A nuestro modo de ver, esta ciencia para todas y todos es una realidad que se va conformando; es decir, hacia la que se va caminando, como ponen de manifiesto la preocupación de los investigadores y los innovadores de la educación científica.

Una vez hemos resaltado cuál es nuestro enfoque respecto de la alfabetización científica, a continuación, nos detendremos en las posibilidades didácticas de las controversias sociocientíficas como medio para impulsar dicha alfabetización.

Estamos convencidos de que las SSI son un recurso muy rico de cara a relacionar la enseñanza y el aprendizaje de determinados conocimientos científicos con aquellas otras consideraciones sociales, política, culturales, etc. que permitan a las y los estudiantes formarse una opinión fundamentada sobre estos temas. Avanzando en la capacidad de argumentar y poder participar con criterio en los temas que les afecten, tanto personal como socialmente; contribuyendo así al ejercicio de una ciudadanía democrática.

\subsection{Acerca de las controversias sociocientíficas.}

Cuando hablamos de controversias socio-científicas a lo que nos referimos es a aquellos problemas o cuestiones sociales que surgen de manera frecuente en nuestra sociedad y que tienen implicaciones científicas y/o tecnológicas (Ariza et al, 2014). Estos problemas sociales relacionados con la ciencia aparecen debido a la compleja relación entre ciencia y sociedad (Díaz y Jiménez-Liso, 2012; Garrido y Couso, 2014). Además, se consideran "problemas" porque no hay un consenso acerca de cómo se podrían resolver. Además, como sostienen diferentes autores (Levinson, 2006; Ratcliffe y Grace, 2003; Sadler, 2011) implican connotaciones éticas y morales. 
En este mismo sentido, Sadler (2004) se refiere a ellas como cuestiones sociales controvertidas con vínculos conceptuales y/o de procedimiento con respecto a la ciencia. Las SSI se caracterizan por ser asuntos que generan conflicto y dividen a la sociedad dando lugar a opiniones contrapuestas, están abiertos y suscitan debate (Legardez y Simonneaux, 2006).

Siguiendo a Jiménez-Aleixandre (2010) las SSI son, como hemos establecido, dilemas o controversias sociales que tienen en su base nociones científicas pero que además se relacionan con otros campos: sociales, éticos, políticos y ambientales. Simonneaux y Simonneaux (2009) las sitúan como cuestiones relacionadas con temas socio-sociológicos, como la globalización, la inmigración o cuestiones sociocientíficas referidas a organismos genéticamente modificados, clonación y móviles. Tienen implicaciones en biología, sociología, ética, política, economía o medio ambiente, etc., y se constituyen cuando las diferencias que se forman entre ellas no se limitan al campo de la ciencia.

En definitiva, hablamos de SSI para referirnos a aquellos problemas relacionados con ciencia y sociedad que denotan la compleja relación que existe entre ambas, y que normalmente surgen cuando existe una discrepancia por intereses de diversa naturaleza entre los diversos actores, fuerzas sociales o entidades civiles que participan en ella (investigadores, científicos, opinión pública, administración, empresas privadas que financian los estudios, etc.). Es decir, además de ser las SSI cuestiones socialmente controvertidas, en cuanto que están socialmente vivas -por ser temas que unen los problemas reales y actuales de la sociedad con un componente científico-, incorporan otras disciplinas e intereses (políticos, económicos, etc.) e implican, a su vez, la evaluación de aspectos relacionados con la cuestión moral y la ética (Evagorou, Jimenez-Aleixandre y Osborne, 2012; Zeidler y Nichols, 2009; Garrido y Couso, 2014).

\subsection{El estudio/inicio de las SSI como "ciencia en construcción".}

Estas controversias o cuestiones sociocientíficas comenzaron a estudiarse dentro del movimiento CTS -del que ya hemos hablando anteriormente-, a finales de los setenta y principios de los ochenta en torno a temas muy dispares: desde la comida a la energía nuclear, y desde la biotecnología al calentamiento global (Musiani, 2010). Actualmente constituyen una gran línea de investigación dentro del área de la Didáctica de las Ciencias Experimentales, como se desprende del alto número de aportaciones realizadas acerca de esta temática realizadas por los investigadores en los últimos congresos del área en el ámbito europeo 4 .

Una de las principales características de las SSI es que están formadas por la "ciencia en construcción" (science in the making); esto es, la ciencia que se está haciendo y que aún no cuenta con un consenso ni por parte de la comunidad científica (que avale su rigurosidad), ni por parte de la sociedad (que entienda su aportación ni sentido). De ahí que algunos autores ofrezcan una definición más amplia que las conceptualiza como aquellos problemas socialmente relevantes que se caracterizan por pretender conectar con el mundo real y ser abiertos, complejos y controvertidos. Esto último como consecuencia de la falta de consenso científico y de la existencia de una respuesta única y definitiva ante el problema (Sadler, Barab y Scott, 2007; Sadler, 2011). Tal y como sostienen dichos autores, de

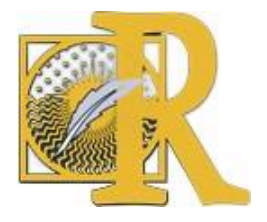


algunos de estos problemas no se tienen respuestas definitivas, y cualquiera que sea la postura o el debate que se tengan, tanto a nivel individual como a nivel social, con respecto a dichos problemas, no le va a ser ajeno a la sociedad; puesto que su importancia irá en aumento a medida que continúen los avances de la ciencia y los problemas derivados de su aplicación.

Mientras que la ciencia que aparece en los libros de texto -"ready-made-science"- indica sólo el producto final derivado de la investigación científica - aquella que sí cuenta con el consenso científico-; hablamos de la "ciencia en construcción" cuando nos referimos a aquella que actualmente se está trabajando en los laboratorios: es la ciencia que está a la vanguardia de la investigación. Comprender las diferencias que existen entre ambas ciencias (ready-made-science y science in making) puede constituir una herramienta importante para entender e interpretar el desacuerdo existente entre los expertos, como algo natural y necesario y que forma parte de la naturaleza de la ciencia; además de ayudarnos a percibir su funcionamiento de una forma más holística.

\subsection{El sentido formativo de las SSI para la ciencia en construcción.}

Las SSI nos permiten estudiar esta ciencia que se está formando, donde se perciben claramente las discrepancias entre científicos y otros agentes sociales, y no sólo aquella ya establecida que aparece en los libros de texto. El planteamiento de diferentes opiniones sobre dilemas sociales que surgen y que están relacionados con la ciencia, se convierten en un punto de partida y en un motor del aprendizaje, de cara a desarrollar la alfabetización científica de toda la población. Por esta razón las últimas propuestas educativas animan a incorporar problemas sociocientíficos y haciendo de ellas el eje de la actividad educativa (Sadler y Zeidler, 2009).

Si el objetivo prioritario de la enseñanza de las ciencias es formar ciudadanas y ciudadanos con las herramientas suficientes como para enfrentarse a los diversos problemas y retos que se advierten en nuestra sociedad actual (Sadler, 2004a; Sadler, 2009a; Sadler, 2011 y Zeidler et al. 2005), la educación basada en SSI debe abordar explícitamente el siguiente desafío: formar a las y los estudiantes para que sean capaces de afrontar con éxito todas aquellas cuestiones relacionadas con la ciencia. Cuestiones que demandan una adecuada alfabetización científica, centrada en la naturaleza de las situaciones con un componente científico. Es decir, aquellas en las que las y los estudiantes van a encontrarse retos y desafíos reales, y en los que tienen lugar consideraciones que van más allá de las cuestiones relacionadas con la ciencia.

Según estos argumentos, las SSI se convierten en un canal privilegiado para explorar la práctica científica, pues es en esta "ciencia en construcción" donde se perciben claramente las discrepancias entre científicos y otros expertos (Kolstø, 2001a). Además presentan una ventaja, ésta es, la de explicitar los procesos sociales que, generalmente, no son visibles para el público -porque se suelen quedar confinados en el laboratorio-, y que, de este modo, son expuestos públicamente (Musiani, 2010). Esto no hace más que mostrar la necesidad de la visión II de la alfabetización científica, y que hemos abordado en el apartado anterior. 
Teniendo en cuenta lo planteado hasta ahora, consideramos que mientras que nos dediquemos a enseñar a las y los estudiantes para que aprendan solo lo relacionado con el contenido científico, -y aunque se les ayude a que participen mejor en las negociaciones y a que tomen mejores decisiones relacionadas con aquellas cuestiones que vinculan ciencia y sociedad-, es poco probable que esta sea la forma más eficiente de apoyar la participación del estudiante en estas cuestiones (Zeidler et al., 2005). Es por ello que proponemos el uso de las SSI para alcanzar la alfabetización científica, puesto que así no solo aprenden datos e informaciones inconexas acerca del conocimiento científico, sino que son también capaces de construir ideas propias y reflexiones sobre los aspectos sociales, económicos, éticos y morales del problema con el que se enfrentan (Sadler, 2009a).

Así pues, desatacamos como durante la última década las SSI se han convertido en un tema importante dentro de la literatura en Didáctica de las Ciencias Experimentales (en adelante DCE). Este movimiento SSI se ha constituido sobre otros enfoques que comparten el objetivo de preparar mejor a las y los estudiantes para participar en discursos y decisiones relacionadas con temas socialmente relevantes y relativos la ciencia, es decir, alcanzar la alfabetización científica. En el siguiente apartado desarrollaremos la evolución que ha tenido este movimiento dentro de la DCE.

\subsection{Desarrollo del movimiento controversias sociocientíficas dentro de la enseñanza de las ciencias.}

El movimiento SSI ha crecido dentro de la DCE con el objetivo de usar estos temas o problemas sociocientíficos como contexto para enseñar ciencias; persiguiendo promover la alfabetización científica y la formación de ciudadanos (Sadler, 2004루 ; Sadler, 2009ạ; Sadler, 2011 y Zeidler et al., 2005). Si promover la ciudadanía es una prioridad para los profesores de ciencias, entonces tendremos que considerar los contextos que creamos para el aprendizaje de la enseñanza de las ciencias; y ahí las SSI juegan un papel fundamental.

Domenech (2014) destaca el uso de SSI en el aula de ciencias como contexto, siendo una de las iniciativas propuestas para orientar la organización del currículum y diseñar actividades, con el objetivo de promover que los alumnos adquieran la competencia o alfabetización científica. Por su parte, Sadler, Barab y Scott (2007) justifican el uso para la enseñanza de las ciencias a través de las SSI, utilizando éstas como contextos para el contenido de la ciencia y para desarrollar la educación ciudadana. Su uso muestra avances en el conocimiento del contenido científico, así como en la comprensión de la naturaleza de la ciencia, ocupándose también de hacer frente a la educación ciudadana en las clases de ciencia. En un sentido parecido, España y Prieto (2009) proponen para favorecer la alfabetización científica utilizar las SSI en el aula de ciencias como un contexto adecuado para contribuir a formar ciudadanos conscientes de los avances científico-tecnológicos y preparados para tomar decisiones responsables; teniendo en cuenta tanto determinados conocimientos científicos como las consideraciones éticas y morales implicadas.

Como se puede apreciar, el enfoque de la literatura que ha surgido en torno a las SSI es variado y diverso. Ya desde el movimiento Ciencia-Tecnología-Sociedad, se le ha prestado mucha atención a las controversias para llevar el debate sociocientífico al aula de ciencias, a la par que se trabajan los objetivos de la alfabetización científica y tecnológica, la responsabilidad social, la toma de decisiones o la educación para la democracia. De una

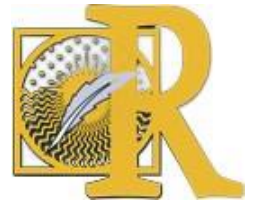


parte, España y Prieto (2010) destacan algunos de los aspectos relacionados con las SSI a los que se les está prestando más atención desde la investigación en DCE como contexto para apreciar la naturaleza de la ciencia, trabajar la argumentación o implicar aspectos morales y afectivos en el aprendizaje de las ciencias (Kolstø, 2001a; Zeidler et al., 2002; Sadler, Chambers y Zeidler, 2004).

Así, podemos afirmar que dentro de la relación SSI y naturaleza de la ciencia a la que estos autores hacen referencia son numerosos los problemas sociocientíficos que implican situaciones en las que se demanda un conocimiento científico que aún no se ha consolidado. Roberts (2007) ya destacó la importancia de la naturaleza de la ciencia situándola dentro de la alfabetización científica y Kolstø (2001aㅡ) apunta que la identificación y utilización didáctica de las SSI puede ayudar a promover una visión de la ciencia más compleja, en la que, como decíamos, los casos de "ciencia terminada" convivan con casos de "ciencia en construcción".

Además, las SSI se han establecido como contextos efectivos para el desarrollo de los conocimientos y procesos que contribuyen a la alfabetización científica, incluyendo la argumentación basada en pruebas, la construcción de consenso, razonamiento moral y comprensión y aplicación del conocimiento del contenido científico (Sadler, 2009ạ; Zeidler, Applebaum y Sadler, 2011).

Como hemos señalado los aspectos morales y afectivos en las SSI también han sido abordados en la literatura (ej. Sadler, 2004b) puesto que en el contexto de la toma de decisiones sobre problemas sociocientíficos, no solo se pone en juego conocimiento científico, sino valores, creencias, actitudes, aspectos morales, aspectos sociales, etc., que guardan relación con la afectividad.

Además de esta clasificación a la que acabamos de hacer referencia, establecida por España y Prieto (2010), acerca de las diferentes investigaciones que se han llevado a cabo sobre SSI, existen otras, como la de Sadler (2011) que distingue entre: estudios que se han centrado en la construcción conceptual con respecto a cómo los enfoques basados en SSI están relacionados con la alfabetización científica, estudios centrados en el razonamiento y en la toma de decisiones en contextos SSI y relaciones entre toma de decisiones en SSI y ciertas comprensiones, como la naturaleza de la ciencia; además de otros trabajos que han explorado temas asociados con la evaluación SSI y resultados relacionados y, por último, el estudio de las SSI como figura en el aula. Pero este autor ya realizó una clasificación previa, (Sadler, 2004aㅜ), acerca de los estudios relacionados con el razonamiento informal respecto a las SSI. Apuntando que la mayoría de los trabajos empíricos con razonamiento informal en SSI abordan cuatro temas principales: a) la argumentación sociocientífica (ej. Jiménez-Aleixandre et al., 2000), b) las relaciones entre la naturaleza de la ciencia (NOS), conceptualizaciones y toma de decisiones sociocientíficas (ej. Erduran y Dagher, 2014; Sadler et al., 2004), c) la evaluación de la información relativa a las cuestiones sociocientíficas (Sadler et al., 2004; Kolstø, 2001b) y d) la influencia de la comprensión conceptual en el razonamiento informal respecto a las SSI (Hogan, 2002), realizando en la mayoría de los casos estudios cualitativos. 
Las distintas clasificaciones realizadas sobre las investigaciones de SSI nos ponen de manifiesto que la literatura existente es muy extensa. En línea con las propuestas de alfabetización científica y tecnológica que se lanzan desde DCE, en investigación se está resaltando el papel que pueden dar los problemas sociocientíficos en las complejas relaciones que existen entre Ciencia-Tecnología-Sociedad con unas características que las hacen muy útiles para crear situaciones ricas para el aprendizaje de aspectos que influyen en nuestras decisiones. En el siguiente epígrafe revisaremos algunas de las últimas propuestas que se están lanzando para trabajar las SSI en el aula en el aula de ciencias.

\section{Repasando propuestas educativas con SSI.}

A lo largo de este artículo hemos ido situando el concepto de controversias sociocientíficas o SSI y hemos establecido cómo éstas constituyen un buen contexto para desarrollar la alfabetización científica de la ciudadanía, ya que permiten a los estudiantes comprender la importancia de la ciencia en la vida cotidiana, profundizar en cómo la gente usa la ciencia y desarrollar la capacidad de ser consumidores críticos de la información científica (Kolstø, 2001a).

Los últimos currículums españoles (LOE y LOMCE5) tanto en educación primaria como en secundaria, están diseñados desde la perspectiva de la alfabetización científica. Y es que hay diversas razones por las que en la sociedad actual es necesaria una ciudadanía científica y tecnológicamente alfabetizada, tal y como apuntan Prieto, España y Martín (2012):

- razones de carácter económico, ya que ningún país se podrá asegurar un desarrollo si no se ejecutan programas educativos que mejoren tanto la alfabetización científica del conjunto de la población como la preparación de científicos y tecnólogos;

- razones de carácter político-social, ya que la ciudadanía debe de tener suficiente cultura científica y técnica para comprender, controlar y participar democráticamente en las decisiones de científicos y tecnólogos;

- razones de carácter cultural, para poder disfrutar del conocimiento científico y tecnológico como parte de nuestra cultura y finalmente de carácter funcional, para podernos desenvolver y actuar en el mudo que nos rodea donde los productos resultados de la ciencia y tecnología están cada vez más presentes.

Pero las SSI, además de constituir un contexto idóneo para el desarrollo de la alfabetización científica, también promueven la participación en la discusión y el debate, proporcionando un marco adecuado para la comprensión de la información científica y de la naturaleza de las ciencias; así como para desarrollar habilidades de pensamiento de orden superior de argumentación y pensamiento crítico (Evagorou et al., 2012; Zeidler y Nichols, 2009). Sin embargo, y a pesar de estos reconocidos beneficios que acabamos de mencionar, las SSI no se incorporan de forma habitual en el aula de ciencias, menos aún en educación primaria en la que a priori parecen suponer un gran reto para maestros y alumnos (Garrido y Couso, 2014).

La revisión bibliográfica realizada en un trabajo previo (Díaz-Moreno y Jiménez-Liso, 2012) nos ha proporcionado un punto de partida para situar qué propuestas didácticas con SSI plantean los autores para las clases de ciencias y cuáles son sus principales finalidades,

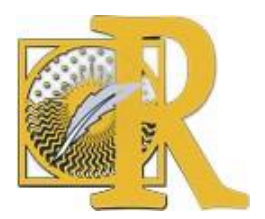


destacando entre ellas de forma mayoritaria la alfabetización científica del alumnado y el fomento de la cultura científica como futuros ciudadanos y ciudadanas (Pedrós, Martínez y Varo 2007; Millar y Hunt 2006; Marco-Stiefel 2003; Marco-Stiefel e lbañez, 2006; GutiérrezJulián, Gómez-Crespo y Martín-Díaz, 2009; Albe, 2006; Marbá, 2010).

Las actividades con SSI propuestas por diferentes autores y recogidas (Tabla 2) muestran una serie elementos comunes en las mismas como son las lecturas y posteriores debates. Además, la mayoría de ellas están diseñadas para implementarlas en el aula de Secundaria (12-18 años), salvo algún ejemplo que se ha desarrollado en aulas Universitarias, reforzando así la idea de Garrido y Couso (2014) antes mencionada acerca de la implementación de secuencias con SSI en otros niveles educativos fuera de la educación secundaria.

\begin{tabular}{|c|c|c|}
\hline $\begin{array}{c}\text { Controversia } \\
\text { (Autores) }\end{array}$ & Actividades propuestas $y / 0$ secuencia de actividades & Etapa \\
\hline $\begin{array}{l}\text { Enriquecimiento } \\
\text { de alimentos } \\
\text { con ácido fólico } \\
\text { Jarman y } \\
\text { McClune (2007) }\end{array}$ & $\begin{array}{l}\text { - Utilización de "cartas al director" para extraer argumentos a favor y en contra sobre } \\
\text { este tema } \\
\text { - Lectura de una carta publicada en el "Belstaf Telegraph" con argumentos en contra } \\
\text { de la fortificación de la harina con ácido fólico. } \\
\text { - Lectura de una carta publicada en el "Belstaf Telegraph" con argumentos a favor de } \\
\text { la fortificación de la harina con ácido fólico. } \\
\text { - Realización de diálogo con toda la clase. Preguntas acerca de las posturas } \\
\text { - Diálogo final acerca de la obligatoriedad o no de la fortificación de la harina con } \\
\text { ácido fólico. }\end{array}$ & Secundaria \\
\hline $\begin{array}{c}\text { Accidente } \\
\text { nuclear de } \\
\text { Chernobyl } \\
\text { Marco-Stiefel } \\
(2006)\end{array}$ & $\begin{array}{l}\text { - Transformar fuentes primarias en fuentes divulgativas. Recursos en red (web de la } \\
\text { BBC "Chernobyl } 20 \text { years") convertidos en material para clase: lecturas, videos, } \\
\text { esquemas, mapas y testimonios. } \\
\text { - Lectura de artículo de "The Guardian" } \\
\text { - Trabajo en el aula también con los contenidos clásicos, ej. Fundamentos de Energía } \\
\text { Nuclear }\end{array}$ & Secundaria \\
\hline $\begin{array}{l}\text { Calentamiento } \\
\text { global. } \\
\text { Simonneaux y } \\
\text { Simonneaux } \\
(2009)\end{array}$ & $\begin{array}{l}\text { - Lectura crítica de textos, argumentación y debate en grupos. } \\
\text { - Lectura crítica de textos (extractos de artículos de la revista "Terre Savage", del } \\
\text { períodico "Sud-Ouest" y de la página web http://www.pyre'nees-pireneus.com) } \\
\text { - Justificación escrita de un posible cambio de opinión después de leer los textos } \\
\text { - Debate en grupo } \\
\text { - Justificación escrita de un posible cambio de opinión después del debate }\end{array}$ & Universidad \\
\hline $\begin{array}{l}\text { Biocombustibles } \\
\text { Escudero, Cid y } \\
\text { Escudero (2009) }\end{array}$ & $\begin{array}{l}\text { - Uso de miniQuest } \\
\text { - Análisis crítico de prensa (Revista Consumer y Diario El País) y campaña publicitaria } \\
\text { (http://www.abengoabioenergy.com/) }\end{array}$ & Secundaria \\
\hline $\begin{array}{l}\text { Efectos de los } \\
\text { teléfonos } \\
\text { móviles sobre la } \\
\text { salud } \\
\text { Albe (2006) }\end{array}$ & $\begin{array}{l}\text { - Introducción al tema mediante la lectura de titulares de periódicos y artículos de } \\
\text { actualidad } \\
\text { - Discusión de los datos científicos aportados. Opiniones del alumnado } \\
\text { - Se le muestra a la clase el funcionamiento de un teléfono móvil } \\
\text { - Realización de un juego de rol en el alumnado Debate con una confrontación } \\
\text { argumental. } \\
\text { - Vuelven a dar su opinión por escrito sobre la peligrosidad en el uso de teléfonos } \\
\text { móviles }\end{array}$ & Secundaria \\
\hline
\end{tabular}

Tabla 2. Adaptado de Díaz-Moreno y Jiménez-Liso (2012).

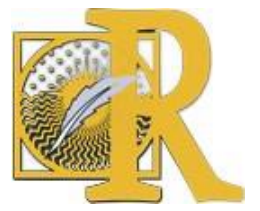


Dada la gran producción de trabajos relativos a las SSI, que ya hemos mencionado, no nos es posible hacer referencia a todas las propuestas que actualmente se están desarrollando por los autores; aun así, sí queremos revisar brevemente algunas de ellas, para lo que hemos seleccionado por el nivel educativo en el que están siendo implementadas.

Existen muy pocas investigaciones en el ámbito de la formación del profesorado o sobre las dificultades para enseñar SSI en el aula y por eso queremos destacar el trabajo de Garrido y Couso (2014). Este trabajo referencia al diseño e implementación de una formación inicial de futuros maestros sobre las controversias sociocientíficas y su enseñanza en el aula de primaria en el marco del proyecto europeo PreSEES (Preparing Science Educators for Everyday Science). Dicho proyecto persigue involucrar a maestros de primaria en formación inicial en discusiones críticas sobre temas actuales de ciencias a través de las SSI y prepararlos para enseñarlas. Dicho objetivo responde al interés por proporcionar oportunidades para que el alumnado experimente la ciencia en contextos análogos a los contextos que se encontrarán fuera de la escuela, con el objetivo de conseguir la alfabetización científica de todos los ciudadanos (Albe, 2007; Sadler y Zeidler, 2009a). Desde esta perspectiva, la formación consistirá en hacer experimentar a los futuros maestros como estudiantes lo que ellos después tendrán que enseñar, y trabajar los aspectos didácticos con propuestas de aula ejemplares con los objetivos de comprender qué son las SSI y reflexionar sobre su enseñanza, así como diseñar e implementar actividades con SSI.

Por último, queremos destacar el proyecto europeo del $7^{\circ}$ Programa Marco PARRISE (Promoting Attainment of Responsible Research and Innovation in Science Education) (Ariza et al., 2014), que intenta promover la alfabetización científica en la sociedad. Para ello PARRISE pretende compartir, refinar y mejorar módulos de desarrollo profesional para el profesorado de ciencias en educación primaria y secundaria integrando dos aproximaciones pedagógicas: el aprendizaje de las ciencias por investigación guiada (IBSE) y las controversias sociocientíficas (SSI); dando así lugar a una nueva aproximación educativa, llamada aprendizaje basado en la investigación de problemas socio-científicos (SSIBL). Dentro de este marco se desarrollarán módulos SSIBL para la formación del profesorado, así como así como la formación inicial y continua del profesorado de educación primaria y secundaria utilizando SSIBL.

A la luz de estos últimos trabajos podemos afirmar que las tendencias más novedosas apuestan por un uso de las SSI en otros niveles educativos, en concreto en formación inicial del profesorado para que posteriormente puedan llevarlo a las aulas de educación primaria, no quedando las SSI relegadas al ámbito de la educación secundaria como apuntaban hasta ahora las propuestas analizadas.

Coincidimos en que el uso de propuestas que trabajan las SSI deben de comenzar en las primeras etapas educativas, acercando así desde edades tempranas ciencia y sociedad al alumnado y promoviendo una educación científica que nos permita formar ciudadanas y ciudadanos capaces de comprender, analizar y discutir sobre SSI; llegando a ser una parte activa en la investigación e innovación responsable, educando ciudadanos críticos y alfabetizados científicamente. 


\section{Conclusiones.}

En este artículo hemos situado el potencial didáctico que presentan las controversias sociocientíficas como herramienta didáctica para el desarrollo de la alfabetización científica. Hemos situado, en primer lugar, la situación de emergencia que presenta la educación científica, así como la necesidad de acortar la brecha existente entre los aprendizajes promovidos por las ciencias en la escuela actualmente y las demandas sociales; o dicho de otra manera, entre educación científica y sociedad. Seguidamente hemos presentado en qué consiste la alfabetización científica y hemos situado las SSI como contexto para alcanzarla y trabajar la educación científica. Esto dado que las SSI proporcionan una situación atractiva de la ciencia pero sobretodo, permiten democratizar la formación del conocimiento, ayudando a nuestro estudiantes a desarrollar esquemas intelectuales auto sostenibles en cuestiones que suelen estar monopolizadas por las autoridades 0 responsables políticos (Zeidler, 2014).

Como hemos podido comprobar a lo largo del desarrollo de este artículo, las SSI están resultando de gran riqueza para la investigación dentro de la enseñanza de las ciencias, ya que permiten que se pongan de manifiesto factores importantes que intervienen en los procesos de enseñanza-aprendizaje (Díaz-Moreno, 2013; Domenech, 2014). Vemos así el gran potencial de las SSI como línea de investigación didáctica y como contexto para la enseñanza de las ciencias que contribuye a facilitar la toma de decisiones sobre el diseño de actividades y su aplicación a través de un enfoque que atiende tanto al desarrollo cognitivo como al pensamiento moral y ético (España y Prieto, 2010); enfoque que, en definitiva, favorece el desarrollo de la alfabetización científica y la formación de ciudadanos. La investigación en el aula ha demostrado que un enfoque centrado en las SSI utilizado para la educación científica se convierte en un proceso de transformación para el alumnado y su profesor/a, produciendo cambios estructurales fundamentales y profundos que reorganizan las normas en un nivel básico de las redes sociales y de la comprensión. La enseñanza SSI es más que una estrategia de enseñanza, favoreciendo el desarrollo de conocimiento de los contenidos y una serie de habilidades y disposiciones, tales como la curiosidad, la resolución de problemas, habilidades de comunicación y colaboración, toma de decisiones y el aprendizaje autodirigido (Zeidler, Applebaum y Sadler, 2011). Por ello las últimas investigaciones apuntan hacia un uso de las SSI fuera del aula de secundaria, instruyendo a futuros maestros en estos problemas durante su formación inicial para que posteriormente sean capaces de llevarlas a cabo en el aula.

Para terminar, hemos de señalar que, a pesar de la importancia de las controversias dentro de las DCE como instrumento para desarrollar la alfabetización científica y la formación de ciudadanos y ciudadanas, la situación actual de España parece tender a eliminar este uso de las controversias para quedarnos con el conocimiento indiscutible. Esto se aprecia bien en el hecho de haber suprimido la asignatura "Educación para la Ciudadanía" y "Ciencias para el mundo contemporáneo"; olvidando con ello que, como señala Martínez-Rodríguez (2012), "el motor del conocimiento científico es precisamente la controversia, el debate, la pluralidad de perspectivas y de enfoques y tendiendo a nueva política educativa que parece querer desterrar el debate y la reflexión". 


\section{Referencias.}

- Aguilar, T. (2001). "Aprendizaje de las ciencias y ejercicio de la ciudadanía". En Membiela, P. (Ed.), Enseñanza de las Ciencias desde la perspectiva Ciencia-TecnologíaSociedad, (77-89), Madrid: Narcea.

- Albe, V. (2006). Tratar controversias científicas contemporáneas en clase. Alambique, 49, 95-104.

- Albe, V. (2007). Students' positions and considerations of scientific evidence about a controversial socioscientific issue. Science Education, 17(8-9), 805-827.

- Ariza, M. R., Abril, A.M., Quesada, A. y García, F.J (2014). Conectar el aprendizaje por investigación con controversias socio-científicas. Contribuciones del proyecto europeo PARRISE. Comunicación presentada en XXVI en Encuentros en Didáctica de las Ciencias Experimentales. Huelva.

- Blanco-García, N. (s/f). ¿Qué conocimiento para qué escuela? Quaderns digitals, Recuperado de: http://www.quadernsdigitals.net/datos web/articles/kikiriki/k39/k39conocimiento.htm

- Blanco-López, A. (2004). Relaciones entre la educación científica y la divulgación de la ciencia. Revista Eureka sobre Enseñanza y Divulgación de las Ciencias, 1(2), 70-86.

- Bybee, R. (1997). Towards an Understanding of Scientific Literacy. En Gräber, W., Bolte, C. (Eds.), Scientific Literacy. Kiel: IPN.

- Castaño, E., Cuello, A., Gutiérrez, N., Rivero, A, Sampedro, C. y Solís, E. (2006). Educación y cultura científica. Sevilla: Junta de Andalucía. Consejería de Educación.

- Couso, D., Jiménez, M. P., López-Ruíz, J., Mans, C., Rodríguez, C., Rodríguez, J. M. y Sanmartí, N. (2011). Informe ENCIENDE. Enseñanza de las Ciencias en la Didáctica escolar para edades tempranas en España. Madrid: Rubes. Recuperado de: http://www.cosce.org/pdf/Informe ENCIENDE.pdf

- DeBoer, G.B. (2000). Scientific literacy: another look at its historical and contemporary meanings and its relationship to science education reform. Journal of Research in Science Teaching, 37(6), 582-601.

- Díaz-Moreno, N. y Jiménez-Liso, M. R. (2012). Las controversias socio-científicas: temáticas e importancia para la educación científica. Revista Eureka sobre Enseñanza y Divulgación de las Ciencias, 9 (1) ,54-70.

- Díaz-Moreno, N. (2013). Determinación de una controversia socio-científica al nivel local; el caso del agua como recurso natural en la prensa almeriense. Tesis Doctoral, Universidad de Almería.

- Domenech, A. (2014). L'ús de les controvèrsies sociocientífiques per promoure la competència científica a l'educació secundària: el cas de la medicalització i el TDA-H. Tesis Doctoral, Universidad Autónoma de Barcelona. Recuperado de: http://ddd.uab.cat/record/127186

- Erduran, S., y Dagher, Z. R. (2014). Reconceptualizing Nature of Science for Science Education. Springer: Netherlands.

- EURYDICE (2011). Science Education in Europe: National Policies, Practices and Research. Brussels: Education, Audiovisual and Culture Executive Agency.

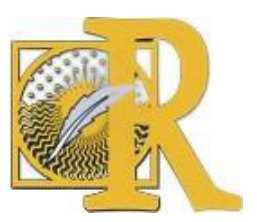


- Escudero, M., Cid, M. y Escudero, R. (2009). La controversia de los agrocombustibles, una propuesta didáctica para las ciencias del mundo contemporáneo. Revista Eureka sobre Enseñanza y Divulgación de las Ciencias, 6, 131-139.

- España, E. y Prieto, T. (2009). Educar para la sostenibilidad: el contexto de los problemas socio-científicos. Revista Eureka sobre Enseñanza y Divulgación de las Ciencias, 6, 345-354.

- España, E. y Prieto, T. (2010). Problemas socio-científicos y enseñanza-aprendizaje de las ciencias. Investigación en la Escuela, 71, 17-24.

- Evagorou, M., Jiménez-Aleixandre, M. P., y Osborne, J. (2012). "Should We Kill the Grey Squirrels?" A Study Exploring Students' Justifications and Decision-Making. International Journal of Science Education, 34(3), 401-428.

- Fourez, G. (2005). Alfabetización científica y tecnológica: acerca de las finalidades de la enseñanza de las ciencias. Buenos Aires: Colihue.

- Fundación Española para la Ciencia y la Tecnología (FECYT) (2011). Percepción social de la ciencia y la tecnología, quinta encuesta. Madrid: FECYT.

- Garrido, A. y Couso, D. (2014). Análisis del aprendizaje y autoeficacia de las controversias socio-científicas (SSI) de futuros maestros de primaria en una formación inicial. Comunicación presentada en XXVI Encuentros de Didáctica de las Ciencias Experimentales. Huelva (España).

- Gutiérrez-Julián, M., Gómez-Crespo, M. y Martín-Díaz, M. (2009). La información científica en la televisión. Enseñanza de las Ciencias, Número Extra VIII Congreso Internacional sobre Investigación en Didácticas de las Ciencias, 1440-1443.

- Hogan, K. (2002). Small groups' ecological reasoning while making an environmental management decision. Journal of Research in Science Teaching, 39, 341-368.

- Jarman, R. y McClune, B. (2007). Developing Scientific Literacy. Using News Media in the Classroom. New York: McGraw-Hill.

- Jiménez, R. (2012). Ayer, hoy y mañana de la investigación en la enseñanza de las ciencias. Comunicación presentada en XXV Encuentros de Didáctica de las Ciencias Experimentales. Santiago de Compostela.

- Jiménez-Aleixandre, M. P. (2010). 10 ideas clave. Competencias en argumentación y uso de pruebas. Barcelona: Graó.

- Jiménez-Aleixandre, M. P., Rodríguez, A. B., y Duschl, R. A. (2000). "Doing the lesson" or "doing science:" Argument in high school genetics. Science Education, 84, 757-792.

- Kolstø, S. D. (2001a). Scientific Literacy for Citizenship: Tools for Dealing with the Science Dimension of Controversial Socioscientific Issues. Science Education, 85(3), 291-310.

- Kolstø, S. D. (2001b). To trust or not to trust,... '-pupils' ways of judging information encountered in a socio-scientific issue. International Journal of Science Education, 23, 877-901.

- Legardez, A. y Simonneaux, L. (2006). L'école à l'épreuve de l'actualité. Enseigner les questions vives. Issy les Moulineaux: ESF.

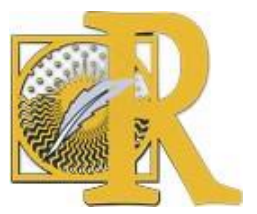

Fecha de recepción: 28-12-2017 Fecha de aceptación: 03-06-2019 didáctica para el desarrollo de la alfabetización científica 
- Levinson, R. (2006). Towards a theoretical framework for teaching controversial socioscientific issues. International Journal of Science Education, 28, 1201-1224.

- Lindsay, S. (2011). Scientific Literacy: A Symbol for Change. En J. Lougrhan, K. Smith, A. Berry (Eds.), Scientific Literacy Under the Microscope (pp. 3-15). Clayton, Australia: Sense.

- Marbá, A. (2010). Aprender ciencias leyendo noticias: un reto para la escuela del siglo XXI. Trabajo presentado en XXIV Encuentros de Didáctica de las Ciencias Experimentales del 21 al 23 de Julio de 2010 en Baeza (Jaén).

- Marco-Stiefel, B., Ibáñez, T. (Coords.) (2006). Las fronteras de la ciencia. Educación científica para la ciudadanía. Materiales didácticos. Madrid: Narcea.

- Marco-Stiefel, B. (2003). La ciencia y la tecnología escolar en el marco de las nuevas alfabetizaciones. Alambique, 38, 21-32.

- Marco-Stiefel, B. (2001). Alfabetización científica y enseñanza de las ciencias. Estado de la cuestión. Enseñanza de las ciencias desde la perspectiva Ciencia-TecnologíaSociedad. Formación científica para la ciudadanía, 33-46.

- Marco-Stiefel, B. (1995). La naturaleza de la ciencia en los enfoques CTS. Alambique, 3, 19-29.

- Martín-Díaz, M. J., Gutiérrez, M. S. y Gómez, M. A. (2011). Las ciencias en la ESO desde la perspectiva de alfabetización científica. En Caamaño, A. (Eds.), Física y Química. Complementos de formación disciplinar (127-149). Barcelona: Grao.

- Martínez-Losada, C. (2010). Contextos formales y no formales de aprendizaje científico en XXIV Encuentros de Didáctica de las Ciencias Experimentales del 21 al 23 de Julio de 2010 en Baeza (Jaén).

- Martínez-Rodríguez, J. B. (2012). Negar al alumnado las controversias sociocientíficas supone robarle la esencia del conocimiento. Blog de ediciones Morata. Recuperado de: http://www.edmorata.es/nuestro-bloc/negar-al-alumnado-las-controversiassociocientificas-supone-robarle-la-esencia-del

- Millar, R. y Hunt, A. (2006). La ciencia divulgativa: una forma diferente de enseñar y aprender ciencia. Alambique, 49, 20-29.

- Musiani, F. (2010). Dalla ricerca all'insegnamento (Il passo é breve). TECNOSCIENZA. Italian Journal of Science and Technology Studies, 1(2), 165-184.

- Organización para la cooperación y el desarrollo económico (OCDE). (2006). PISA 2006 marco de la evaluación: conocimientos y habilidades en ciencias, matemáticas y lectura. Recuperado de: http://www.oecd.org/dataoecd/59/2/39732471.pdf

- Pedrinaci, E., Caamaño, A., Cañal, P. y De Pro, A. (2012). 11 ideas clave. El desarrollo de la competencia científica. Barcelona: Graó.

- Pedrós, G. Martínez, M.P. y Varo, M. (2007). La sección cartas al editor: Un planteamiento científico y social en didáctica de las ciencias. Enseñanza de las Ciencias, 25(2), 195-204.

- Prieto, T., España, E. y Martín, C. (2012). Algunas cuestiones relevantes en la enseñanza de las ciencias en una perspectiva Ciencia-Tecnología-Sociedad. Revista Eureka sobre Enseñanza y Divulgación de las Ciencias, 9(1), 71-77.

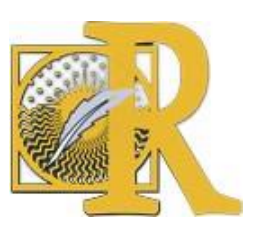

Fecha de recepción: 28-12-2017 Fecha de aceptación: 03-06-2019

Díaz-Moreno, N., Caparrós-Martín, E., Sierra-Nieto, E. (2019). Las controversias sociocientíficas como herramienta didáctica para el desarrollo de la alfabetización científica 
- Ratcliffe, M. y Grace, M. (2003). Science Education for Citizenship. Teaching SocioScientific Issues. Maidenhead: Open University Press.

- Roberts, D. A. (2007). Scientific Literacy/ Science Literacy. En Abell, S. K. y Lederman, N. G. (Eds.). Handbook research on science education (729-780). Mahwah, New Jersey: Lawrence Erlbaum.

- Sadler, T. D. (2011). Socio-scientific Issues in the Classroom: Teaching, Learning and Research. Netherlands: Springer.

- Sadler, T. D. (2009a). Socioscientific issues in science education: labels, reasoning, and transfer. Cultural Studies in Science Education, 4, 697-703.

- Sadler, T. D. (2009b). Situated learning in science education: socio-scientific issues as contexts for practice. Studies in Science Education, 45(1), 1-42.

- Sadler, T. D. y Zeidler, D. L. (2009). Scientific Literacy, PISA, and Socioscientific Discourse: Assessment for Progressive Aims of Science Education. Journal of Research in Science Teaching, 46(8), 909-921.

- Sadler, T. D., Barab, S. A. y Scott, B. (2007). What do students gain by engaging in socioscientific inquiry? Research in Science Education, 37(4), 371-391.

- Sadler, T. D. (2004a). Informal reasoning regarding socioscientific issues: A critical review of research. Journal of Research in Science Teaching, 41, 513-536.

- Sadler, T. D. (2004b). Moral sensitivity and its contribution to the resolution of socioscientific issues. Journal of Moral Education, 33 (3), 339-358.

- Sadler, T. D., Chambers, F. W. y Zeidler, D. L. (2004). Students' conceptualization of the nature of science in response to a socioscientific issue. International Journal of Science Education, 26(4), 387-409.

- Shamos, A. (1995). The Myth of Scientific Literacy. New Brundswick, Nueva Jersey: Rutgers University Press.

- Simonneaux, L. y Simonneaux, J. (2009). Students' socio-scientific reasoning on controversies from the viewpoint of Education for Sustainable Development. Cultural Studies in Science Education, 4, 657-687

- Solbes, J. y Torres, N. (2012). Análisis de competencias de pensamiento crítico desde el aborde de las cuestiones sociocientíficas: un estudio en el ámbito universitario. Didáctica de las Ciencias Experimentales y Sociales, 26, 247-269.

- Zeidler D. L. (2014). "Socioscientific Issues as a Curriculum Emphasis: Theory, Research and Practice". En Lederman, N.G y Abell, S.K. (Eds.) Handbook of Research on Science Education II (697-726). Routledge.

- Zeidler, D. L., Applebaum, S. M. y Sadler, T. D. (2011). "Enacting a socioscientific issues classroom: Transformative transformations". En T. D. Sadler (Ed.) Socio-scientific issues in science classrooms: Teaching, learning and research (277-306). Netherlands: Springer.

- Zeidler, D. L., y Nichols, B. H. (2009). Socioscientific Issues: Theory and Practice. Journal of Elementary Science Education, 21(2), 49-58.

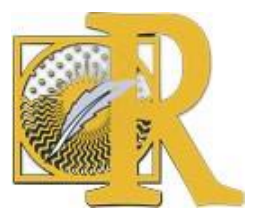

Fecha de recepción: 28-12-2017 Fecha de aceptación: 03-06-2019 
- Zeidler, D. L., Sadler, T. D., Simmons, M. L. y Howes, E. V. (2005). Beyond STS: A research-based framework for socio-scientific issues education. Science Education, 89, 357-377.

- Zeidler, D. L., Walker, K. A., Ackett, W. A. y Simmons, M. L. (2002). Tangled up in views: Belief in the nature of science and responses to socio-scientific dilemmas. Science Education, 86, 343-367.

${ }^{1}$ El Informe Rocard (2007), auspiciado por la Comisión Europea, analiza la educación científica, ofreciendo datos y propuestas sobre la base de las cuales mejorar, precisamente, la enseñanza de las ciencias en las etapas de Educación Infantil y Primaria.

2 Manifiesto pedagógico "No es verdad", disponible en: http://www.uclm.es/seminarios/sehisp/archivos master/pedromiralles/NO\%20ES\%20VERDAD.pdf

${ }^{3}$ Se puede consultar en: http://pus.sagepub.com

${ }^{4}$ Un ejemplo muy representativo de este auge lo encontramos en el espacio de discusión e intercambio científico que significan las conferencias organizadas por ESERA (European Science Education Research Association. http://www.esera.org).

${ }^{5}$ Ley Orgánica 2/2006 de 3 de mayo de Educación (LOE), promulgada en el año 2006. Fue sustituida parcialmente por la Ley Orgánica 8/2013 de 9 de diciembre para la mejora de la calidad del sistema educativo (LOMCE).

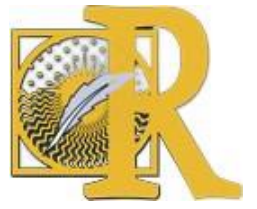

